\title{
Salendo sulla Vedetta. Un rilievo laser scanner all'interno del castello di Gallipoli
}

Going up on the lookout. A laser scanner survey inside the Gallipoli castle

\author{
Giovanna Muscatello $^{a}$, Aurora Quarta ${ }^{b}$, Carmine Mitello $^{c}$ \\ ${ }^{a}$ Università del Salento, Lecce, Italy, giovannamuscatello@libero.it \\ ${ }^{\mathrm{b}}$ Università del Salento, Lecce, Italy, aurora.quarta@gmail.com \\ ${ }^{\mathrm{c}}$ Musa s.r.l., Giurdignano (Le), Italy, c.mitello@tiscali.it
}

\begin{abstract}
Gallipoli is a city located along the Ionian coast of Salento (Apulia, Italy) and it still preserves its original defensive system. Nowadays, the phases dating from the sixteenth century are visible together with the later ones and, in recent years, particular attention was dedicated to the Castle. It was opened for visitors from 2014, except to the so-called tower "Della Vedetta", located on the northwestern quadrilateral's corner.

The only accessible areas of the castle's lower level are two rooms with a connecting corridor, but the lower portion of the examined tower offers a rare proof of graffiti that has few local parallels. After a deep bibliographic and archivist research and the comparison with analogue works on similar well-studied contexts, we have carried out a direct analysis of this unknown portion through a 3D laser scanner. The scanner result proved to be an excellent tool to investigate the tower, whose articulation is made up of different rooms located on different levels connected by flights of stairs and where numerous mouths of fire are set. This 3D model allows to delineate the internal articulation, the constructive technique, the structural interventions and the whole corpus of graffiti too. The 3D survey helped to extrapolate considerable and various data. Moreover, it has suggested that a realization of a complete 3D model of the Castle can be a fruitful resource for its knowledge and it could represent a precious tool for an interactive enjoyment of the sectors still not open to visitors.
\end{abstract}

Keywords: Castel of Gallipoli, Torre della Vedetta, naval graffiti, digital mapping.

\section{Introduzione}

La città di Gallipoli è ubicata sulla costa ionica salentina: il territorio abitato ha la caratteristica di essere diviso in due parti collegate da un ponte, la cosiddetta "Città Vecchia", centro storico che sorge su uno scoglio ed un tempo più alto rispetto ai $14 \mathrm{~m}$ attuali poiché interessato da un lentissimo bradisismo positivo, ed il "Borgo Nuovo", costituito in conseguenza dell' aumento demografico sempre più crescente e si sviluppa verso l'entroterra in lunghezza per circa $3 \mathrm{~km}$ ed è in continua espansione
(Saladini, 1987; Quarta, 2015). A nord del centro storico vi è l'area del nuovo porto commerciale, mentre a sud ed in prossimità del castello vi è quello ancora oggi definito l'antico porto: in entrambi i casi, si tratta di insenature riparate che permettevano e permettono ancora oggi un valido riparo per le imbarcazioni e, soprattutto per la protezione dell'antico porto (nel "Mar Piccolo o del Canneto"), in cui il castello aveva un ruolo fondamentale. 


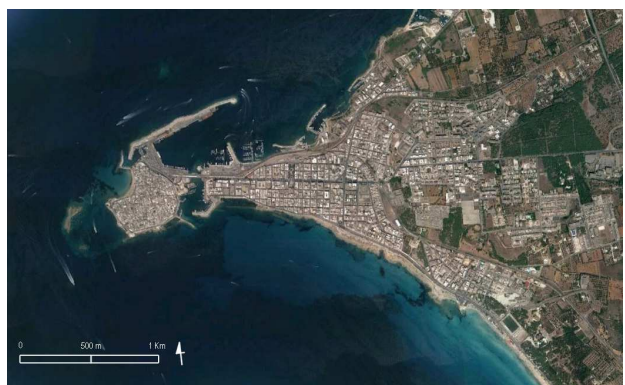

Fig. 1. Il centro abitato di Gallipoli (Google Earth).

Il sistema di difesa di Gallipoli è parte integrante di un più vasto apparato che già nel tratto di costa, compreso tra Taranto e Santa Maria di Leuca, presenta una fitta catena di torri comunicanti tra loro: a seguito dell'assedio di Otranto per mano turca nel 1480 e delle successive e continue minacce lungo la costa (Morciano, 1543; Gallipoli, 1544), la necessità di proteggere il territorio diviene infatti, una reale priorità (Quarta, 2017).

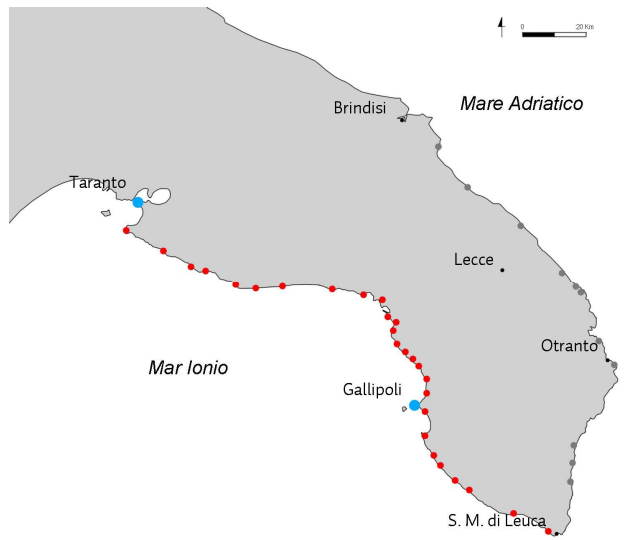

Fig. 2. Fascia ionica salentina con il sistema di torri costiere superstiti e castelli (Quarta 2017).

La difesa della cittadina ionica quindi, era articolata in una cinta muraria con 12 capisaldi (di cui se ne conservano 8) ed un castello, risultato di numerosi rimaneggiamenti ed aggiustamenti avvenuti nel corso dei secoli.

La struttura si articola su una planimetria quadrangolare sui cui quattro spigoli vi sono i torrioni, una torre poligonale e di maggiori dimensioni, detta anche Torre Grande, e due circolari (il torrione di sud-ovest è crollato nel XVIII secolo). Sul lato orientale sopravvive il Rivellino, un tempo addossato al castello (Bacile di Castiglione, 1915; Vita, 1984).

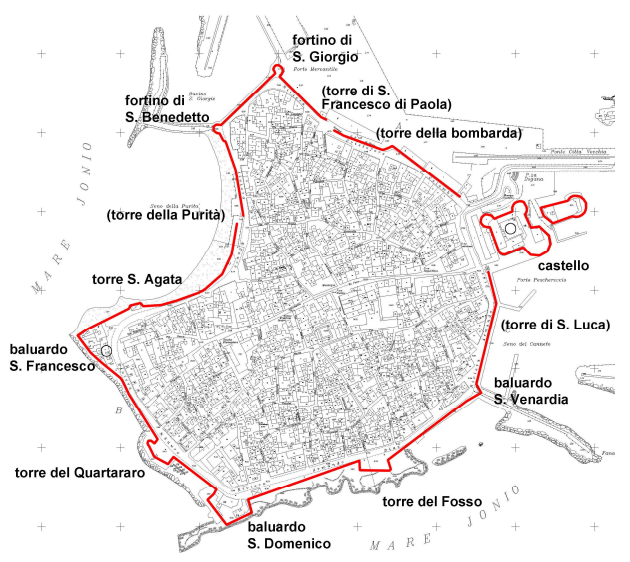

Fig. 3. Sistema difensivo della città (Quarta 2017).

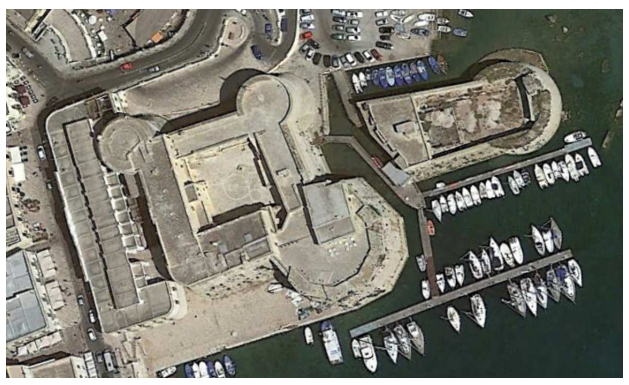

Fig. 4. Il castello di Gallipoli (Muscatello-QuartaMitello, 2018).

\section{La Torre della Vedetta}

Il Torrione della Vedetta è ubicato sullo spigolo nord-occidentale del quadrilatero del castello: non è possibile avere una visione complessiva di esso poiché è in parte inglobato nell'edificio dell'ex-Mercato Pubblico. Ha una base circolare con un diametro di $20 \mathrm{~m}$ alla quale segue un primo toro, una scarpata di circa $7 \mathrm{~m}$, un secondo toro marcapiano da cui si prosegue verticalmente per circa $4 \mathrm{~m}$ sino al coronamento di archetti ciechi e beccatelli che sorregge un tamburo di $5 \mathrm{~m}$ di altezza massiccio e sporgente (Vernole 1933). 


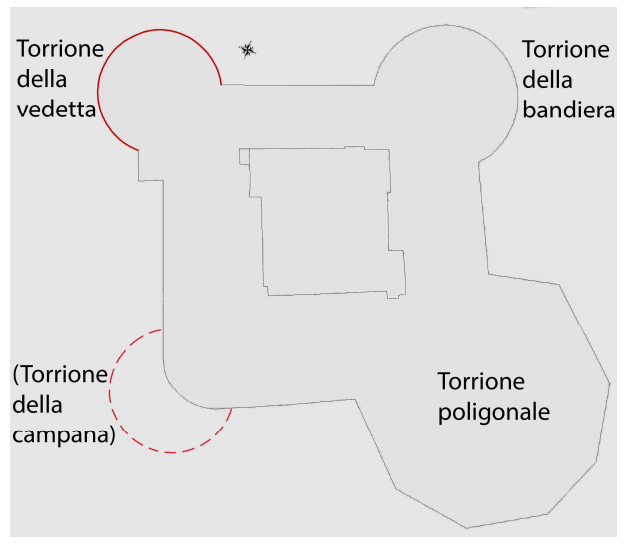

Fig. 5. Castello di Gallipoli, planimetria. In rosso l'ubicazione della Torre della Vedetta.

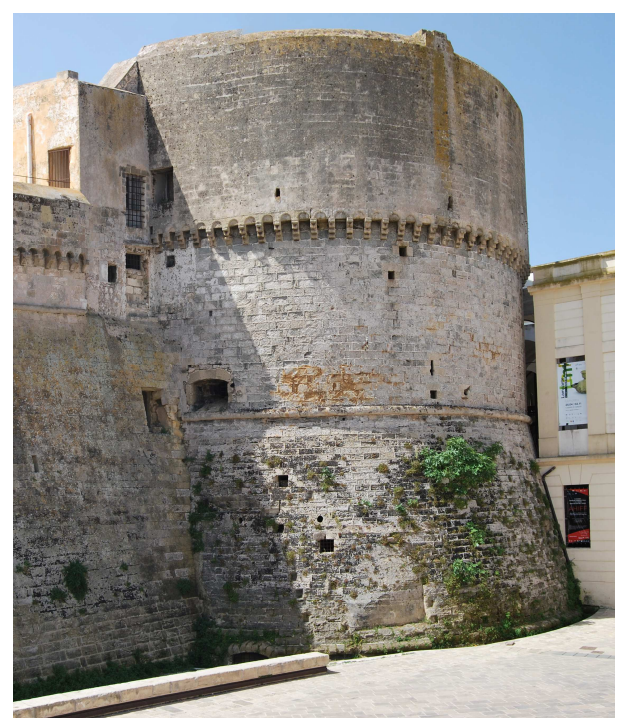

Fig. 6. Il Torrione della Vedetta, lato nord.

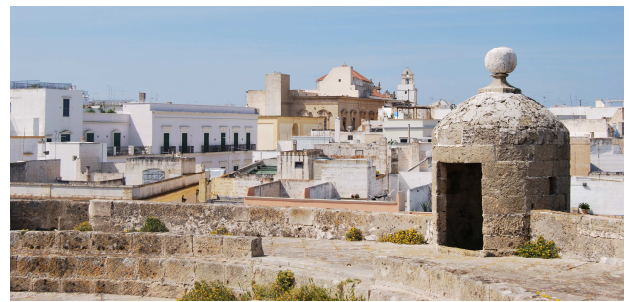

Fig. 7. Garitta di vedetta; sullo sfondo, la basilica concattedrale, centro dell' isola.
Sulla sommità del torrione inoltre, si conserva integralmente la garitta di vedetta da cui, appunto, prende il nome, in direzione della città.

L'interno della torre è raggiungibile dalle terrazze del castello: si accede ad un primo vano rettangolare che conduce all'interno del torrione caratterizzato da un'ampia sala con una volta a cupola, su cui sono visibili i fori da ponteggio, che ingloba uno spigolo di una struttura costituito da due murature verticali attualmente intonacate. Il pavimento della sala è coperto da un tavolaccio che probabilmente copre una pavimentazione ormai dissestata.

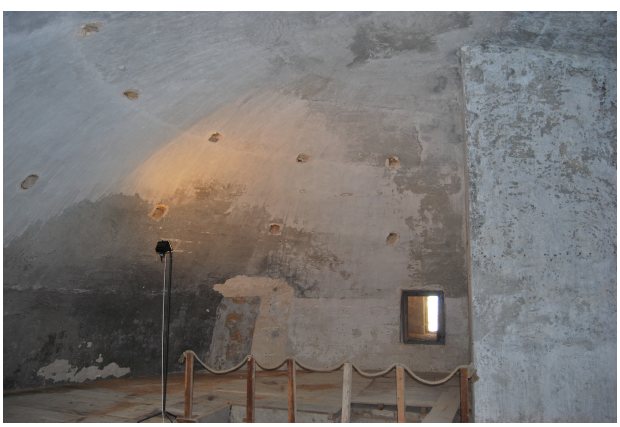

Fig. 8. Interno del torrione: volta a cupola del livello superiore.

Dal livello superiore si accede, attraverso una serie di scale ben articolate all'interno del vano, al livello intermedio fino a giungere al livello 0 (quota di ingresso al castello): qui è visibile una aula con mezza copertura a cupola e diverse feritoie occluse.

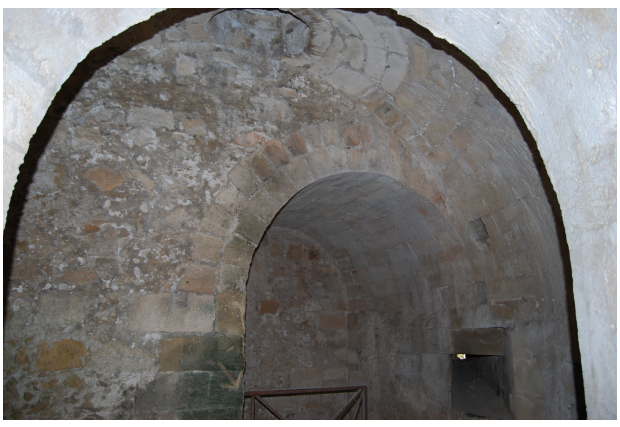

Fig. 9. Interno torrione: livello 0. 
Proseguendo la discesa, si giunge al livello inferiore in cui si apre uno scenario incredibile in cui compaiono segni e raffigurazioni graffite sull'intonaco delle pareti: i soggetti rappresentati variano, oltre alle numerose imbarcazioni anche di grandi dimensioni in percentuale maggiore, vi sono alcune iscrizioni, cerchi con tacche lungo la circonferenza (forse dei "conteggi”), una tabula lusoria, un profilo umano con un caratteristico copricapo, una mano ed una croce con estremità svasate (Muscatello, Quarta, Mitello, 2018).

\subsection{I graffiti navali: rilievo e mappatura digi- tale}

Il presente studio si inserisce in un più ampio processo di conoscenza dell'intero organismo architettonico, attraverso una serie di azioni volte alla comprensione delle fasi storiche e di tutte le modifiche di carattere strutturale che si sono avvicendate nei secoli e che hanno fortemente caratterizzato il castello di Gallipoli. Base imprescindibile del lavoro è stata l'esecuzione del rilievo metrico diretto ed indiretto dell'intera struttura, comprese anche quelle parti ignote ai molti e non facilmente raggiungibili. In quest'ottica rientra l'indagine condotta nella Torre della Vedetta, ancora non fruibile, che è stata rilevata metricamente anche attraverso l'uso della tecnologia laser scanner 3D ad alta definizione a colori che ha permesso di comprendere meglio la morfologia strutturale e gli aspetti dimensionali del complesso. Inoltre, da un'attenta analisi e mappatura delle superfici murarie verticali, è stato possibile registrare la presenza di una serie di raffigurazioni graffite che rappresentano un unicum all'interno del panorama salentino. E' tutt'ora in corso la mappatura digitale dell'intero corpus di graffiti che costituirà un fondamentale catalogo digitale fruibile anche da remoto. Quello che più colpisce tra la varietà dei graffiti, per quantità e per qualità del dettaglio nella rappresentazione, sono le incisioni riproducenti le imbarcazioni, tra cui caracche, galee, sciabecchi e galeazze (Fig. 10).

Tali incisioni risulterebbero successive alla prima metà del XVI secolo e sulla base del differente tratto stilistico e della posizione all'interno della torre, si può certamente affermare che $\mathrm{i}$ graffiti furono realizzati da autori differenti e in periodi differenti. La presenza di graffiti di imbarcazioni rappresenta un'utile strumento, oltre che per l'analisi dell'evoluzione delle costruzioni navali e dei tipi utilizzati nel passato, anche per lo studio socio-economico delle comunità in cui questi vengono ritrovati. Infatti, essi testimoniano gli intensi traffici marittimi che hanno fortemente caratterizzato nei secoli il porto della città di Gallipoli.

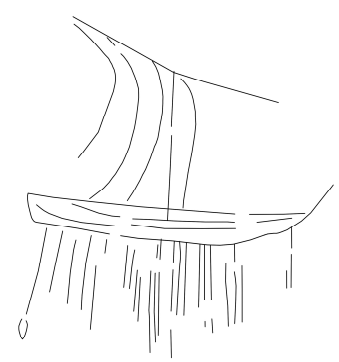

Fig. 10. Torre della Vedetta, livello inferiore. Imbarcazione a vela graffita (Quarta 2018).

Dai dettagli raffigurati nei graffiti si può ipotizzare la frequentazione della torre da parte di persone che conoscevano bene gli elementi costitutivi delle imbarcazioni e che probabilmente operavano nel settore navale o nel suo indotto. $\mathrm{La}$ lettura e lo studio di alcuni graffiti fa propendere verso un'attribuzione di tipo devozionale, quali raffigurazioni ex voto lasciati da uomini legati all'ambiente marittimo. L'interpretazione come ex voto sembrerebbe valere per la raffigurazione, sopra la prua di un'imbarcazione di cui sopravvivono solo poche tracce, di una probabile figura mariana riconoscibile dal volto, con corona e tonaca, secondo il modello iconografico tipico del XVII secolo (a destra in Fig. 11). Nel livello inferiore, tra le varie incisioni è stata identificata un'iscrizione su intonaco dal probabile carattere devozionale che può essere collocata cronologicamente al periodo rinascimentale, accanto alla quale è rappresentata un'imbarcazione graffita (possibile galea) (Fig. 12). Entrambi i graffiti potrebbero essere la testimonianza di un ringraziamento alla divinità per la buona riuscita della navigazione. Tra le rappresentazioni navali ritratte sui muri, si erge maestosa quella graffita sull'intonaco della parete 
della rampa di scale che dal livello inferiore conduce al livello intermedio. Salendo i primi gradini, a sinistra, è raffigurata una probabile galeazza, di notevoli dimensioni, insieme ad altre imbarcazioni più piccole (Figg. 13 e 14). Si tratta della riproduzione di una nave a vele spiegate, uno dei più grandi graffiti navali inediti identifi- cati nel territorio salentino. Eseguito con pregevole accuratezza è alto circa $1,45 \mathrm{~m}$ ed ha una lunghezza di circa $1,70 \mathrm{~m}$. E' plausibile, per le caratteristiche riscontrate, che possa trattarsi di una galeazza, rappresentata all'interno di una scena che racconta un evento che si è compiuto nelle acque gallipoline.

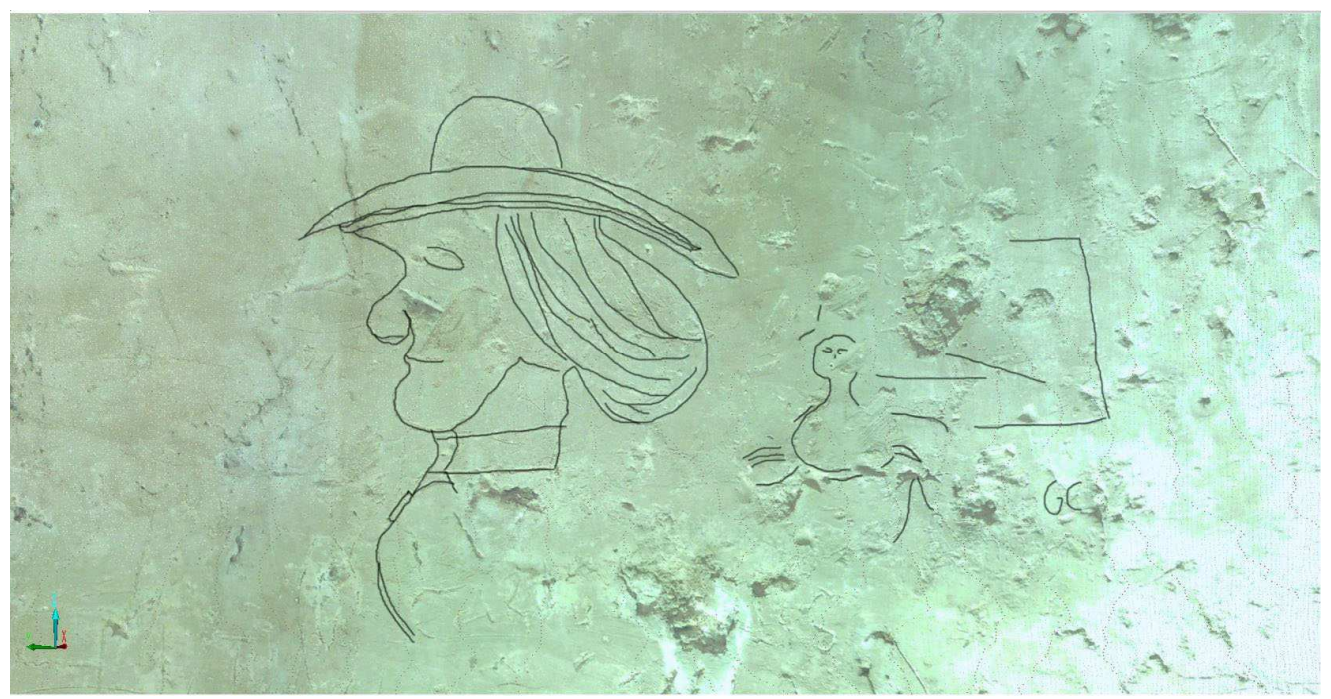

Fig. 11. Torre della Vedetta, livello inferiore. Mappatura digitale da scansione laser. Incisioni graffite lungo le pareti del camminamento sotterraneo. A sinistra, singolare figura umana con cappello. A destra, probabile figura mariana con tracce di imbarcazione (Mitello, Muscatello, 2019).

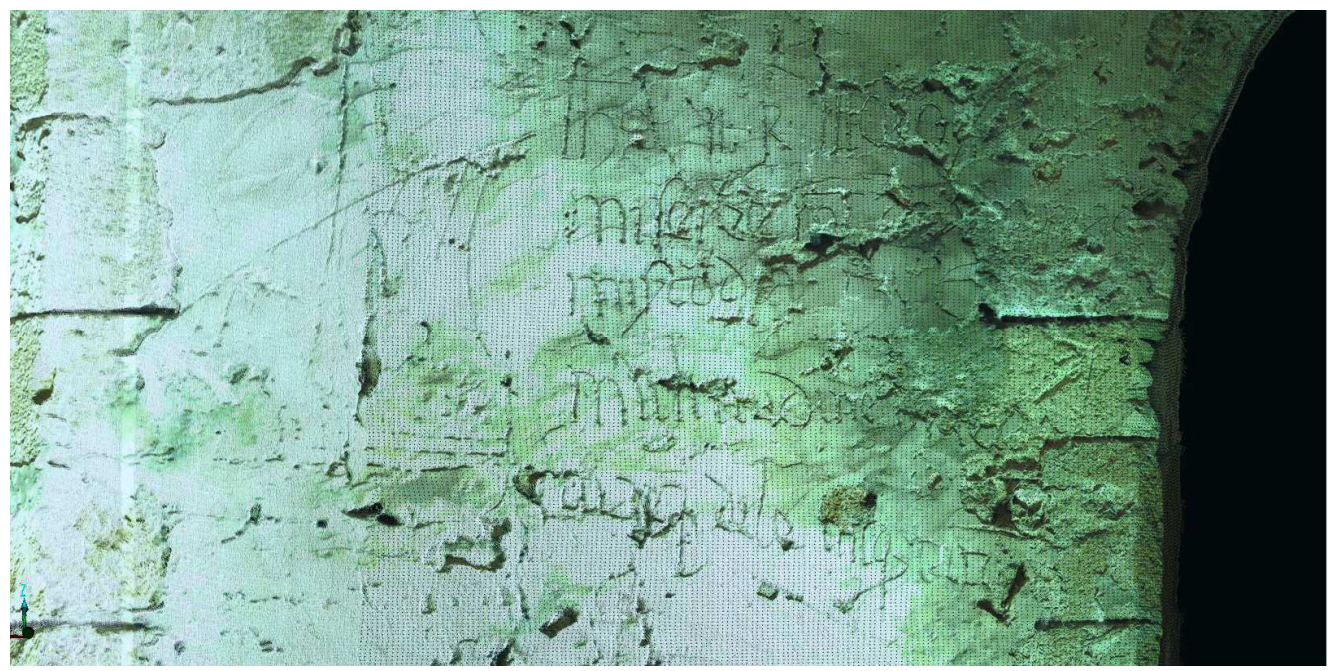

Fig. 12. Torre della Vedetta, livello inferiore. Iscrizione ed imbarcazione graffita. Mappatura digitale da scansione laser con alterazione cromatica (Mitello, Muscatello, 2019). 


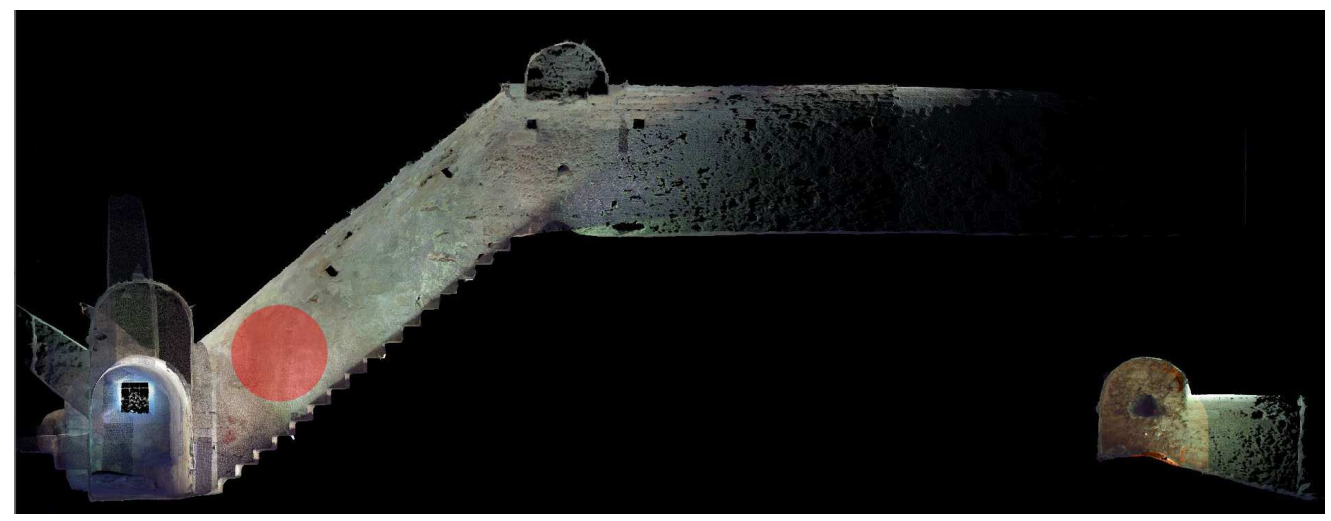

Fig. 13. Torre della Vedetta. Rilievo laser scanner 3D. In evidenza la sezione della scala che dal livello inferiore conduce ad uno dei livelli intermedi. In rosso l'area in cui è presente la grande nave incisa (Mitello, Muscatello, 2019).

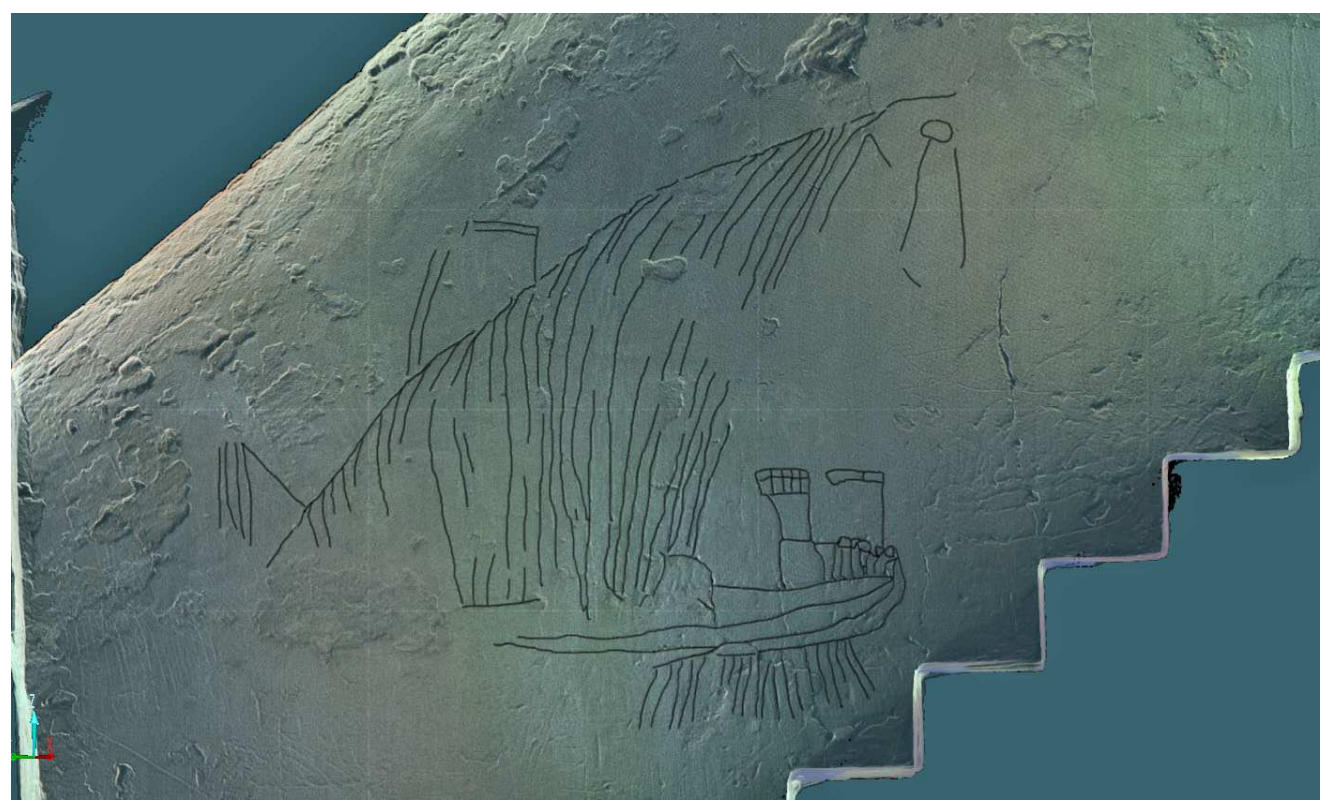

Fig. 14. Torre della Vedetta. Elaborazione tridimensionale della sezione della scale da rilievo laser scanner 3D e mappatura della grande imbarcazione incisa presente sulle pareti, probabile galeazza. Il graffito navale è alto $1,45 \mathrm{~m}$ circa ed è lungo 1,70 m circa (Mitello, Muscatello, 2019).

Per quanto concerne la struttura dell'imbarcazione è ben riconoscibile a poppa il cassero ed il castello per gli ufficiali, mentre purtroppo della prua non abbiamo dati, non si è conservata a causa dell'ammaloramento dell'intonaco su quel lato, per la presenza di umidità che ne ha facilitato il distacco dalle murature.

La cospicua presenza di incisioni navali rispecchia il ruolo strategico di Gallipoli che ha rap- presentato, da un punto di vista commerciale, un crocevia di importanti traffici marittimi. Gallipoli nel XV secolo ricopriva un ruolo commerciale importante, divenne emporio e polo nevralgico da cui far transitare moltissime merci e derrate alimentari. Ebbe contatti con Venezia, Genova, Firenze e con i porti che si affacciavano nel Mediterraneo. Nel XVI e nel XVII secolo l'olio era il principale prodotto di esportazione verso $\mathrm{Ve}$ - 
nezia e l'Oriente. Centinaia di navi hanno solcato il mare gallipolino ed i graffiti rappresentano un'istantanea di un peculiare paragrafo di storia della città.

\section{Conclusioni}

I graffiti della Torre della Vedetta rappresentano un enorme patrimonio storico-artistico, ma a causa della condizione climatica presente all'interno degli ambienti rischiano di essere calcellati per sempre e con essi la storia che si narra nelle decine di rappresentazioni incise, frutto delle esperienze e del pensiero umano. La mappatura digitale in corso e la conseguente catalo- gazione permette di registrare in maniera definitiva le rappresentazioni graffite per renderle visibili alla collettività e disponibili allo studio degli addetti al settore.

\section{Note}

Il rilievo laser scanner 3D della Torre della Vedetta e la mappatura digitale dei graffiti è stata realizzata da Carmine Mitello e Giovanna $\mathrm{Mu}-$ scatello. Le immagini nel testo sono di proprietà degli autori.

Paragrafi 1 e 2: Aurora Quarta. Paragrafi 2.1 e 3: Carmine Mitello e Giovanna Muscatello.

\section{Bibliography}

Bacile di Castiglione, G. (1915). Il castello di Gallipoli. Rassegna Tecnica Pugliese, 5, Bari.

Cossa, A. (2017). Navigando sui muri. Igraffiti navali del Salento (XII-XVII secolo), Botanica Ornamentale Ed., Grottaglie.

Muscatello, G.; Quarta, A.; Mitello, C. (2018). "I sotterranei del castello di Otranto e di Gallipoli: dal rilievo laser scanner 3D all'analisi strutturale", in Marotta, A.; Spallone, R. eds., Defensive Architecture of the Mediterranean, Politecnico di Torino, Torino, vol. IX, pp. 1021-1028.

Quarta, A. (2015). Il castello di Gallipoli: analisi storica e architettonica, Tesi di Specializzazione, Università del Salento, Scuola di Specializzazione in beni archeologici, Salencto.

Quarta, A. (2017, in corso di pubbl.). "The castle of Gallipoli in the defensive system of the Ionian coast in the kingdom of Naples", in Rencontres Internationales du Patrimoine Architectural Méditerranéen (RIPAM), 7, Genova.

Saladini. C.M. (1987). "Gallipoli”, in Zeri, F. ed., Storia dell'arte italiana, inchiesta sui centri minori, Torino, vol. VIII.

Vernole, E. (1933). Il castello di Gallipoli: illustrazione storica architettonica, Roma.

Vita, R. De. (1984). Castelli, torri ed opere fortificate di Puglia, Adda Editore, Bari. 
